\title{
Intra- and Inter-individual genetic variation in human ribosomal RNAs
}

5

\section{Artem Babaian}

10 Terry Fox Laboratory, British Columbia Cancer Agency, Vancouver, BC, Canada. Department of Medical Genetics, University of British Columbia, Vancouver, BC, Canada.

Artem Babaian

Terry Fox Laboratory

BC Cancer Agency

25675 West $10^{\text {th }}$ Avenue

Vancouver, BC, V5Z1L3, Canada

Email: ababaian@bccrc.ca 


\section{Abstract}

The ribosome is an ancient RNA-protein complex essential for translating

30 DNA to protein. At its core are the ribosomal RNAs (rRNAs), the most abundant RNA in the cell. To support high levels of transcription, repetitive arrays of ribosomal DNA (rDNA) are necessary and the long-standing hypothesis is that they undergo sequence homogenization towards rDNA uniformity.

Here I present evidence of the rich genetic diversity in human rDNA, both

35 within and between individuals. Using state-of-the-art genome sequencing data revealed an average of 192.7 intra-individual variants, including some deeply penetrating the rDNA copies, such as the bi-allelically expressed 28S.59A>G. From 104 diverse genomes, 947 high-confidence variants were identified and unmask a hidden genetic diversity of humans.

These findings support the emerging concept that ribosomes are heterogeneous within cells and extends the heterogeneity into the realm of population genetics. Fundamentally, do our ribosomal variants determine how our cells interpret the genome?

Keywords: Ribosomal Variation, Ribosomal Heterogeneity, Ribosomal DNA, Ribosomes, Human Variation 


\section{Introduction}

The ribosome is an RNA-protein complex essential to all life. This ancient complex translates genetic information into protein. In humans, a mature ribosome contains four structural and catalytic RNA molecules: $18 \mathrm{~S}$ in the small subunit and $5 S, 5.8 S$ and $28 \mathrm{~S}$ in the large subunit.

Ribosomes are highly abundant in cells with ribosomal RNA (rRNA) making up $>80 \%$ of the RNA in a human cell.(1) To be transcribed to such a high level, the DNA encoding for rRNA (rDNA) is present at $\sim 600$ copies per diploid genome. The RNA45S gene encodes the 45S pre-rRNA, which is processed and modified into 18S, $5.8 \mathrm{~S}$ and $28 \mathrm{~S}$. The $13.5 \mathrm{~kb}$ RNA45S gene sits within a $43 \mathrm{~kb}$ rDNA repeat,

60 which in turn are arranged in tandem arrays making up the acrocentric arms of chromosomes 13, 14, 15, 21 and 22. The 5S rRNA gene, RNA5S, is found on a tandem-repeated locus at chromosome 1p42.13.(2)

In the initial human genome sequencing project rDNA-containing BACmids were systematically depleted since sequencing was expensive and these

65 repetitive sequences are thought to 'homogenize' into a uniform sequence.(3) Yet notably, in the original research yielding the reference human rDNA, Kuo and colleagues repeatedly cloned and sequenced variants both within and between individuals. $(4,5)$ The variation in these clones localized to 'variable loop regions' and at the time it would have been challenging to distinguish these variants from pseudogenized rDNA. With recent improvements in sequencing technology, rDNA variation has been increasingly characterized in a diverse collection of species(610), so rRNA may not be as homogeneous as previously thought. 
Originally I hypothesized that a transposable element could exist within human rDNA (similar to the Drosophila R1 and R2 elements(11)) yet would be

75 undetected by standard variant analysis of the human genome. I interrogated human rDNA next-generation sequencing data and 're-discovered' massive intraand inter-individual sequence heterogeneity in human rDNA, including expressed variants of $18 \mathrm{~S}$ and $28 \mathrm{~S}$.

\section{Results}

80 Intra-individual variation in rDNA

To measure the intra-individual variation in rDNA, high coverage PCR-free whole genome sequencing data(12) (DNAseq) from the Utah CEPH-1463 trio (daughter, NA12878; father, NA12891; mother, NA12892) was aligned to a reference human rDNA sequence, hgrl (see materials and methods and Figure $851 \mathrm{~A})$.

hgrl was covered to an average depth of $8853 \times$ (Figure 1B). There are an average of $192.7(+-14.3)$ variants per individual at an intra-individual variant allele frequency (iVAF) of greater than $2 \%$. With a $10 \%$ iVAF cut-off, there are 85.3 (+- 4.2) variants per individual (Figure 1C). The distribution of the variants is

90 uneven over the different regions of rDNA (Figure 1D). Excluding $5 \mathrm{~S}$ and $5.8 \mathrm{~S}$ which were invariable, the density of variation was lower in $18 \mathrm{~S}$ and $28 \mathrm{~S}$ at 9.7 variants per kilobase $(\mathrm{kb})$ compared to the other transcribed regions at 15.8 variants per kb, a trend consistent with rDNA variation in other species. $(8,10)$

As expected, the evolutionary conservation of the mature rRNA variants were 95 at evolutionarily variable regions, such as the expansion loops, or were rare within 
individuals. The outlier to this trend is the $28 \mathrm{~S} .59 \mathrm{G}>\mathrm{A}$ variation, which is both evolutionarily conserved and highly variable within individuals. (Figure 1D).

To test if the variants in the mature rRNA are expressed, poly-A selected RNA sequencing (RNAseq) from the ymphoblastoid cell line GM12878 (derived from

100 NA12878) was analyzed.(13) Variants at positions of moderate GC-content could be analyzed such as 28S.59G >A which shows bi-allelic expression. To interrogate a purer rRNA library, K562 total RNAseq (not poly-A selected and not rRNAdepleted) was analyzed with $70.3 \%$ of all reads mapping to rDNA, and here too, 28S.59G >A shows bi-allelic expression (Figure 1E).

While inspecting RNAseq libraries, one variant was recurrently observed at the highly conserved 18S.1248T, yet was absent from DNAseq. In the reference RNA, this base is a uradine, but in the mature $18 \mathrm{~S}$ rRNA, this base is hypermodified to 1-methyl-3-( $\alpha$-amino- $\alpha$-carboxyl-propyl) Pseudouridine (Figure 1E) for its function in the catalyitic core of the ribosome's P-site. This hyper-modification

110 hinders reverse transcriptase function(14), thus the "variation" is likely an error profile specific to this modified nucleotide. Besides being interesting, these findings indicate that most standard poly-A selected RNAseq libraries have 'contaminating' levels of rRNA which can be used to measure variant rRNA expression in future studies.

\section{Inter-individual variation in rDNA}

To measure if there is rDNA variation between individuals, an additional 104 genomes (four genomes from each of 26 populations)(15) were aligned to hgrl. 
Combined variant discovery (see materials and methods) from all 107 genomes yielded 947 variants at 926 distinct positions passing quality control ( $p$

$120<0.001$ ) (Figure 2A, Supplementary File 1 ). $78 \%$ of variants are single base substitutions favoring GC base changes and the remaining are near-equally divided between short insertions and deletions (Figure 2B).

The density of variation across RNA45S largely matches the CEPH-1463 trio distribution but offers finer resolution of regions absent of variation, notably $5 \mathrm{~S}$

125 and $5.8 \mathrm{~S}$, and regions of $18 \mathrm{~S}$ and $28 \mathrm{~S}$. It is important to note that regions of extreme GC-content had lower sequencing coverage across the DNAseq libraries, and more 'no data' points then moderate-GC regions, something which can be addressed with deeper sequencing libraries in the future.

Reasoning that biologically relevant variants, with respect to cell- or tissue-

130 heterogeneous ribosomes, would be maintained in humans, I quantitatively assessed the distribution of the variants in the population (Figure $2 \mathrm{C}$ ). There is a cluster of 23 variants which are both, prevalent in the population, and abundant within individuals (population variant-detection frequency (pVDF) $>0.5$, iVAF 0.33 - 0.66). Alternatively these variants could have had a higher iVAF in human

135 ancestors, and drift could explain their prevalence and abundance as well. To truly measure balancing selection in rDNA, a more complete understanding of its heredity is necessary, but these 23 candidate variants warrant priority biochemical and molecular analysis (Table 1). 
Table 1: Prevalent and abundant rDNA variants

\begin{tabular}{|c|c|c|c|c|c|c|c|}
\hline $\begin{array}{l}\text { Coordinates } \\
\text { (hgr1:) }\end{array}$ & Region & \begin{tabular}{|l} 
Reference \\
Allele
\end{tabular} & \begin{tabular}{|l} 
Variant \\
Alleles
\end{tabular} & iVAF & sd(iVAF) & pVDF & $\begin{array}{l}\text { Variant } \\
\text { Calls (N) }\end{array}$ \\
\hline 1000777 & 5' ETS & CGG & $\begin{array}{l}\text { C, CGGG, } \\
\text { CGGGG }\end{array}$ & 0.52 & 0.29 & 0.99 & 94 \\
\hline 1001683 & 5' ETS & G & GT & 0.37 & 0.19 & 0.88 & 83 \\
\hline 1001756 & 5' ETS & C & $\mathrm{T}$ & 0.45 & 0.17 & 0.84 & 77 \\
\hline 1001944 & 5' ETS & $G$ & C, CGGG & 0.42 & 0.26 & 0.95 & 97 \\
\hline 1002492 & 5' ETS & TGCG & $\mathrm{T}$ & 0.64 & 0.22 & 0.95 & 74 \\
\hline 1003053 & 5' ETS & C & CG & 0.48 & 0.23 & 0.85 & 51 \\
\hline 1003545 & 5' ETS & $\mathrm{C}(\mathrm{CCGT}) 2$ & $\mathrm{C}(\mathrm{CCGT}) 1$ & 0.4 & 0.19 & 0.76 & 71 \\
\hline 1005721 & 5' ETS & $G$ & $A$ & 0.42 & 0.16 & 0.81 & 83 \\
\hline 1006297 & ITS 1 & C & A, CTGA & 0.38 & 0.26 & 0.83 & 60 \\
\hline 1007183 & ITS 2 & (CCGT)1 & (CCGT)2 & 0.42 & 0.19 & 0.93 & 80 \\
\hline 1007812 & ITS 2 & $\mathrm{~T}$ & C & 0.39 & 0.2 & 0.91 & 72 \\
\hline 1007825 & ITS 2 & C & - & 0.38 & 0.17 & 0.95 & 76 \\
\hline 1008007 & 28S.59 & G & A & 0.39 & 0.17 & 0.99 & 106 \\
\hline 1010121 & $28 S .2173$ & G & GC & 0.34 & 0.32 & 0.77 & 82 \\
\hline 1010123 & $28 S .2175$ & G & $\begin{array}{l}\text { T, GGT, } \\
\text { GCGGGGGT }\end{array}$ & 0.59 & 0.25 & 0.79 & 84 \\
\hline 1010134 & 28S.2186 & $\mathrm{T}$ & - & 0.37 & 0.15 & 0.68 & 54 \\
\hline 1010142 & 28S.2194 & GCC & $\mathrm{GC}, \mathrm{G}$ & 0.64 & 0.21 & 0.72 & 51 \\
\hline 1010988 & $28 S .3040$ & A & $\mathrm{G}$ & 0.33 & 0.24 & 0.52 & 40 \\
\hline 1010997 & 28S.3049 & C & $\begin{array}{l}\text { ССТ, } \\
\text { ССТСТСТ }\end{array}$ & 0.43 & 0.18 & 0.89 & 95 \\
\hline 1011025 & 28S.3077 & C & $\mathrm{T}$ & 0.37 & 0.23 & 0.56 & 59 \\
\hline 1011106 & 28S.3158 & C & G, - & 0.44 & 0.33 & 0.61 & 30 \\
\hline 1013136 & 3' ETS & $\mathrm{T}$ & $\mathrm{G}, \mathrm{TG}$ & 0.51 & 0.27 & 0.8 & 78 \\
\hline 1013422 & IGS & C & $\mathrm{T}$ & 0.56 & 0.24 & 0.96 & 75 \\
\hline
\end{tabular}

(Prevalent, pVDF > 0.5; Abundant, $0.33>i V A F>0.66$ )

Surprisingly, by organizing the 26 human populations into their five super-

140 populations, blocks of called variants or even possibly alternative haplotypes emerged (yellow arrows, Figure 2A). These population-uncommon (low pVDF) but intra-individual abundant (higher iVAF) variants (Figure 2C) lends support to the idea that besides intra-individual heterogeneity, there is population-level heterogeneity in rRNA. 
Altogether, there is substantial intra- and inter-individual variation in human rDNA, and these exciting findings raise far more questions then they answer.

\section{Discussion}

The idea that ribosomes are homogenous is increasingly being challenged. $(16,17)$ In a land-mark study, knock-out of the murine large sub-unit ribosomal

150 protein (RP) RPL38 resulted in the specific loss of translation of a set of internal ribosomal entry site-dependent HOX genes, but not in global translation. $(18,19)$ In a biochemical analysis of RPs in mouse embryonic stem cells, the stoichiometry of the different RPs varies between isolated monosomes and polysomes.(20) At the transcriptomic level, the relative RP mRNA in yeast varies with growth conditions,

155 and human RP mRNA varies across tissues,(21) and between normal and malignant cells.(22)

These studies have interrogated ribosomal variation at the protein subunit and post-transcriptional modification levels.(23) In contrast, the intra-individual genetic variation, especially variants present across the populations such as

$16028 \mathrm{~S} .59 \mathrm{G}>\mathrm{A}$, offers an enticing new dimension to intra-cellular or intra-individual ribosomal heterogeneity. Since the 'backbone' of the ribosome is the rRNA, it's tempting to speculate that differences in the RP-composition and rRNA modifications arise from distinct paralogs of RNA45S.

The central role of the ribosome to all translation implies that even small

165 changes to translational efficiency or regulation may have large phenotypic consequences. The paramount biological significance of these genes is 
underscored by how little we know about their variation in humans or other mammals.

It's relatively straightforward to include rDNA analysis to existing high-

170 throughput DNA- and RNAseq analysis as demonstrated, but further elucidation of how to meaningfully interpret these variants from a population and evolutionary perspective is necessary.

In particular there is a broad class of human genetic diseases called ribosomeopathies, such as Diamond-Blackfan anemia and X-linked dyskeratosis

175 congenita, caused by mutation to ribosomal protein or ribosomal biogenesis factors (reviewed by Nakhoul et al,(24)). Notably, there are no known genetic diseases caused by mutation or variants in nuclear rRNA, but this may be due to the previous perceived difficulty in studying rRNA genes. Yet classical linkageanalysis of an inherited aminoglycodside-induced deafness pedigree led to the

180 identification of a causative mutation in the the $12 \mathrm{~S}$ mitochondrial rRNA gene, MTRNR1.(25)

Intra- and inter-individual heterogeneity of human ribosomal RNA raises fundamental questions about translational variation at three levels; differences amongst individuals or populations in a species; differences between tissues; and

185 differences between each individual ribosome within cells. Ultimately, this study scratches the surface of this topic but to facilitate rapid and open exploration of the consequences of these findings, my laboratory notebooks, data and scripts/methods are unrestrictedly available online (http://rRNA.ca). I invite 
bioRxiv preprint doi: https://doi.org/10.1101/118760; this version posted March 21, 2017. The copyright holder for this preprint (which was not certified by peer review) is the author/funder, who has granted bioRxiv a license to display the preprint in perpetuity. It is made available under aCC-BY 4.0 International license.

interested scientists to cooperate in understanding the full implications of this

190 variation in rRNA and the impact this has on human health.

To date I haven't found evidence for a human rDNA-specific transposable element such as R1- or R2. 


\section{Materials and Methods}

An electronic laboratory notebook is available online (http://rRNA.ca) with line-by-line annotated scripts and methods to replicate these experiments in their entirety.

Reference Genomes and rDNA repeats

The $43 \mathrm{~kb}$ reference rDNA repeat, U13369.1 was downloaded from NCBI. The

200 first nucleotide of this sequence is the transcription start site $(+1)$ of the $13.5 \mathrm{~kb}$ $45 \mathrm{~S}$ pre-rRNA, and the last $1 \mathrm{~kb}$ is the rDNA upstream promoter region. Initial analyses were hindered by the sequence complexity of the intergenic spacer, so this study focuses on the genic portion of rDNA. PCR-free whole genome sequencing data from CEPH-1463 was aligned to RNA45S and its promoter as well

205 as a single copy of the 5S locus from hg38 (chr1:228744112-228746352). The sequences were manually edited to match the consensus supported by the data, with the reasoning that this represents the common rDNA sequence at each position. Three difficult to align regions were masked with ' $\mathrm{N}$ ' nucleotides, an AluY element and low complexity sequence in the $5 \mathrm{~S}$ locus and a simple-repeat

210 sequence in the ITS1. The resulting sequence, hgr1, was used in subsequent analysis \{Sequence Accession Pending\}.

Datasets

A complete list of sequencing libraries used in this study is available in Supplementary Table 1. Four genomes from each of the 26 human populations

215 were chosen randomly from the 1000 genomes project data(15). I required paired- 
end Illumina sequencing with a minimum of 20 million sequenced fragments per library. On average the libraries used had 67.8 million sequenced fragments.

The evolutionary Shannon information for each mature rRNA base is based on a mutliple-sequence alignment of rRNA sequences from all domains of life(26) and

220 downloaded from the RiboVision suite.(27)

\section{Alignment}

Initial alignment parameters were selected to maximize sensitivity. DNAseq alignment was performed with bowtie2 (28) using the command `bowtie2 --verysensitive-local -x hgr1 $-1<$ read1.fq.gz> $-2<$ read2.fq.gz> . The majority of the

225 alignments were ran on Amazon EC2 using an C4.2xlarge instance. A publicly available instance image with the necessary software and reference genomes is available: 'crown-161229'.

\section{Variant Discovery}

The purpose of this study was to define the major, high frequency variants in

230 rDNA. Pseudogenized rDNA is a potential source of error in this study, but I reasoned that pseudo-rDNA mutations at identical positions between copies would be rare relative to total rDNA copy number. For variant-discovery, the 107 hgrlaligned bam files were analyzed with the Genome Analysis Toolkit (v.3.6) HaplotypeCaller (29) using standard parameters. HaplotypeCaller performs local

235 de-novo assembly over variant positions, which makes it well suited for genotyping rDNA variants. The 926 called variants had an alternative allele quality score of $>30$ PHRED $(p<0.001)$ and 849 of those variants had a score of 
$>100$ PHRED $\left(p<10^{-10}\right)$. The type of substitutions and indel size distribution was plotted with bcftools.(30)

This analysis should be interpreted as a conservative, lower bound on rDNA variation. With a larger data-set and more importantly, deeper and less PCR-bias genome sequencing, multi-ploidy variant analysis should yield a more complete set of variants at lower intra-individual variant allele frequencies.

Measurements of Variant Allele Frequencies

VCF files were parsed into $R$ with $\operatorname{vcfR}(31)$ and population statistics calculated with custom scripts. For the interrogated variant positions, intraindividual reference allele frequency was calculated by the number of reference genotype read coverage, divided by the total read coverage used in genotyping. The inverse of the reference allele frequency was defined as the intra-individual

250 variant allele frequency (iVAF). I calculated iVAF in this way to account for positions with multiple variant alleles and to measure iVAF in genomes where variant alleles may not have been called by the software, yet variant reads were present. The mean intra-individual variant allele frequency is simply the average value of iVAF for each genome in which a variant was present. many individuals in the population have a measurable variant at a particular position (iVAF $>0.02$ ), divided by the total number of individuals in which that position was sequenced. This normalizes for uneven sequencing coverage in the genomes, especially at high-GC regions. 
260 The product of iVAF and pVDF therefore, is variant allele frequency in the classical sense, taking into account the pool of all rDNA alleles. Although, this makes the assumption that rDNA copy number is the same in each individual which may not be true. $(2,32)$ As such I have refrained from terming any of the variation polymorphisms even though a sub-set (Table 1 ) are most likely

265 polymorphisms.

\section{Acknowledgements}

I'd like to thank my supervisor Dr. Dixie Mager. Thanks to Katharina Rothe, Andrew Chapman, Peter Hurley, Chris Laver and Davide Pellacani for their helpful discussions and critical reviews of the manuscript.

270 I was supported by a studentship award from the Natural Sciences and Engineering Research Council of Canada. Computing resources were a kind gift from hackseq. 


\section{References}

1. Adiconis X, Borges-Rivera D, Satija R, DeLuca DS, Busby MA, Berlin AM, et al. Comprehensive comparative analysis of RNA sequencing methods for degraded or low input samples. Nat Methods. 2013 Jul;10(7):623-9.

2. Stults DM, Killen MW, Pierce HH, Pierce AJ. Genomic architecture and inheritance of human ribosomal RNA gene clusters. Genome Res. 2008 Jan;18(1):13-8.

3. Consortium IHGS. Finishing the euchromatic sequence of the human genome. Nature. 2004 Oct 21;431(7011):931-45.

4. Leffers $\mathrm{H}$, Andersen $\mathrm{AH}$. The sequence of $28 \mathrm{~S}$ ribosomal RNA varies within and between human cell lines. Nucleic Acids Res. 1993 Mar 25;21(6):1449-55.

5. Kuo BA, Gonzalez IL, Gillespie DA, Sylvester JE. Human ribosomal RNA variants from a single individual and their expression in different tissues. Nucleic Acids Res. 1996 Dec 1;24(23):4817-24.

6. Jensen S, Frost P, Torsvik VL. The nonrandom microheterogeneity of $16 \mathrm{~S}$ rRNA genes in Vibrio splendidus may reflect adaptation to versatile lifestyles. FEMS Microbiol Lett. 2009 May;294(2):207-15.

7. Matyášek R, Renny-Byfield S, Fulneček J, Macas J, Grandbastien M-A, Nichols $R$, et al. Next generation sequencing analysis reveals a relationship between rDNA unit diversity and locus number in Nicotiana diploids. BMC Genomics. 2012 Dec 23;13:722.

8. Bik HM, Fournier D, Sung W, Bergeron RD, Thomas WK. Intra-Genomic Variation in the Ribosomal Repeats of Nematodes. PLOS ONE. 2013 Oct 11;8(10):e78230.

9. Chen J, Miao X, Xu M, He J, Xie Y, Wu X, et al. Intra-Genomic Heterogeneity in 16S rRNA Genes in Strictly Anaerobic Clinical Isolates from Periodontal Abscesses. PLoS ONE [Internet]. 2015 Jun 23 [cited 2017 Mar 17];10(6). Available from: http://www.ncbi.nlm.nih.gov/pmc/articles/PMC4477887/

10. Wang W, Ma L, Becher H, Garcia S, Kovarikova A, Leitch IJ, et al. Astonishing 35S rDNA diversity in the gymnosperm species Cycas revoluta Thunb. Chromosoma. 2016;125(4):683-99.

11. Eickbush DG, Eickbush TH. R2 and R2/R1 hybrid non-autonomous retrotransposons derived by internal deletions of full-length elements. Mob DNA. 2012;3:10.

12. Eberle MA, Fritzilas E, Krusche $P$, Källberg M, Moore BL, Bekritsky MA, et al. A reference data set of 5.4 million phased human variants validated by genetic 
inheritance from sequencing a three-generation 17-member pedigree. Genome Res. 2017 Jan 1;27(1):157-64.

13. ENCODE Project Consortium. An integrated encyclopedia of DNA elements in the human genome. Nature. 2012 Sep 6;489(7414):57-74.

14. Maden BE, Corbett ME, Heeney PA, Pugh K, Ajuh PM. Classical and novel approaches to the detection and localization of the numerous modified nucleotides in eukaryotic ribosomal RNA. Biochimie. 1995;77(1-2):22-9.

15. The 1000 Genomes Project Consortium. A global reference for human genetic variation. Nature. 2015 Oct 1;526(7571):68-74.

16. Shi Z, Barna M. Translating the genome in time and space: specialized ribosomes, RNA regulons, and RNA-binding proteins. Annu Rev Cell Dev Biol. 2015;31:31-54.

17. Dinman JD. Pathways to Specialized Ribosomes: The Brussels Lecture. J Mol Biol. 2016 May 22;428(10 Pt B):2186-94.

18. Kondrashov N, Pusic A, Stumpf CR, Shimizu K, Hsieh AC, Xue S, et al. Ribosome-mediated specificity in Hox mRNA translation and vertebrate tissue patterning. Cell. 2011 Apr 29;145(3):383-97.

19. Xue S, Tian S, Fujii K, Kladwang W, Das R, Barna M. RNA regulons in Hox 5' UTRs confer ribosome specificity to gene regulation. Nature. 2015 Jan 1;517(7532):33-8.

20. Slavov N, Semrau S, Airoldi E, Budnik B, van Oudenaarden A. Differential Stoichiometry among Core Ribosomal Proteins. Cell Rep. 2015 Oct 22;13(5):865-73.

21. Yadav A, Radhakrishnan A, Panda A, Singh A, Sinha H, Bhanot G. The Modular Adaptive Ribosome. PLOS ONE. 2016 Nov 3;11(11):e0166021.

22. Guimaraes JC, Zavolan M. Patterns of ribosomal protein expression specify normal and malignant human cells. Genome Biol [Internet]. 2016 Nov 24 [cited 2017 Mar 17];17. Available from: http://www.ncbi.nlm.nih.gov/pmc/articles/PMC5123215/

23. Krogh N, Jansson MD, Häfner SJ, Tehler D, Birkedal U, Christensen-Dalsgaard $M$, et al. Profiling of 2'-O-Me in human rRNA reveals a subset of fractionally modified positions and provides evidence for ribosome heterogeneity. Nucleic Acids Res. 2016 Sep 19;44(16):7884-95.

24. Nakhoul H, Ke J, Zhou X, Liao W, Zeng SX, Lu H. Ribosomopathies: Mechanisms of Disease. Clin Med Insights Blood Disord. 2014 Aug 14;7:7-16. 
25. Prezant TR, Agapian JV, BohIman MC, Bu X, Öztas S, Qiu W-Q, et al. Mitochondrial ribosomal RNA mutation associated with both antibioticinduced and non-syndromic deafness. Nat Genet. 1993 Jul;4(3):289-94.

26. Petrov AS, Bernier CR, Hsiao C, Norris AM, Kovacs NA, Waterbury CC, et al. Evolution of the ribosome at atomic resolution. Proc Natl Acad Sci U S A. 2014 Jul 15;111(28):10251-6.

27. Bernier CR, Petrov AS, Waterbury CC, Jett J, Li F, Freil LE, et al. RiboVision suite for visualization and analysis of ribosomes. Faraday Discuss. 2014;169:195207.

28. Langmead B, Salzberg SL. Fast gapped-read alignment with Bowtie 2. Nat Methods. 2012 Apr;9(4):357-9.

29. McKenna A, Hanna M, Banks E, Sivachenko A, Cibulskis K, Kernytsky A, et al. The Genome Analysis Toolkit: a MapReduce framework for analyzing nextgeneration DNA sequencing data. Genome Res. 2010 Sep;20(9):1297-303.

30. Li H. A statistical framework for SNP calling, mutation discovery, association mapping and population genetical parameter estimation from sequencing data. Bioinforma Oxf Engl. 2011 Nov 1;27(21):2987-93.

31. Knaus BJ, Grunwald NJ. VcfR: an R package to manipulate and visualize VCF format data. bioRxiv. 2016 Feb 26;041277.

32. Gibbons JG, Branco AT, Godinho SA, Yu S, Lemos B. Concerted copy number variation balances ribosomal DNA dosage in human and mouse genomes. Proc Natl Acad Sci U S A. 2015 Feb 24;112(8):2485-90. 


\section{A}

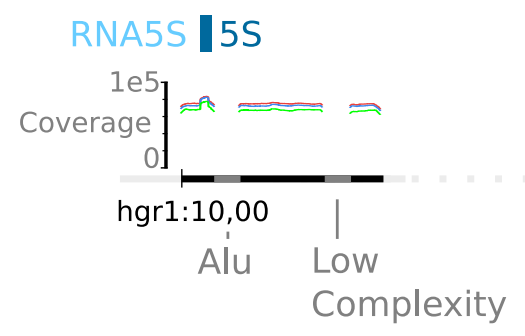

B

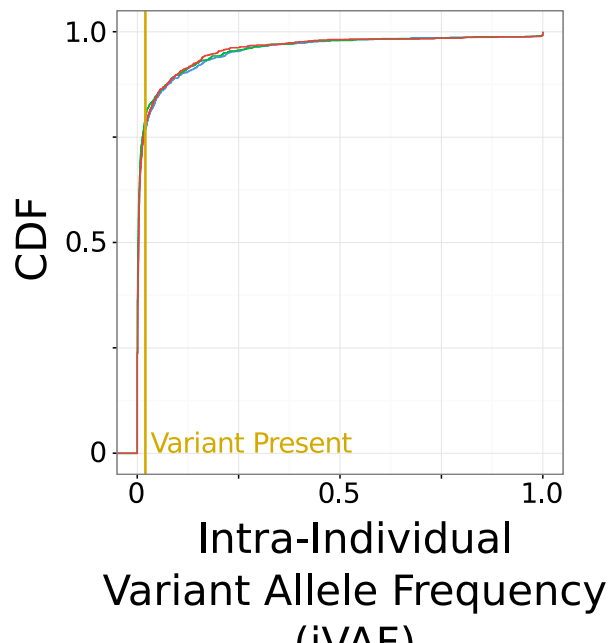

$\mathrm{D}$

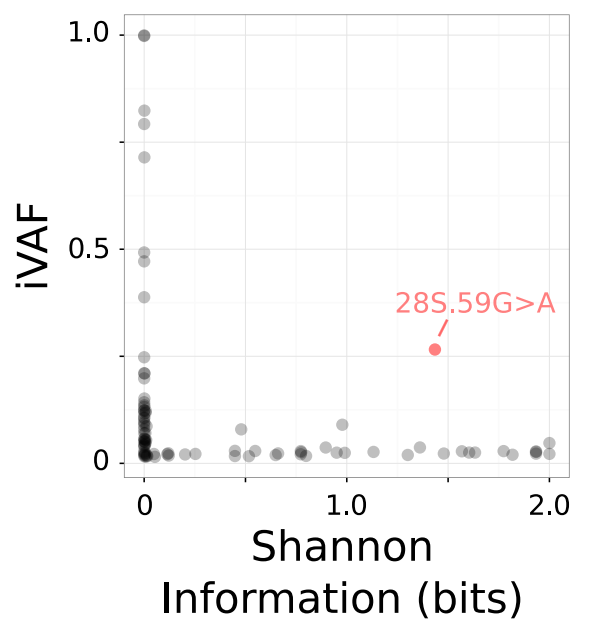

$\mathrm{E}$

\section{NA12878 \\ DNAseq}

GM12878

RNAseq

K562

Total RNAseq
C

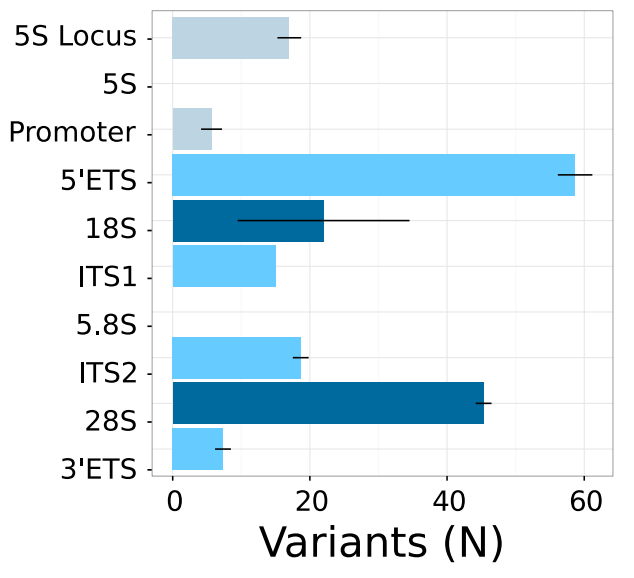

(iVAF)

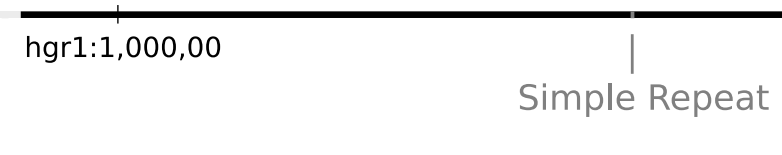

285 3' ETS

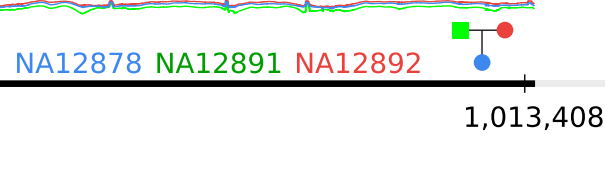




\section{Figure 1: Intra-individual variation at the rDNA locus}

A) Map of the hgrl reference sequence used in this study. It includes a single repeat of the entire RNA5S locus and RNA45S with adjacent sequences. In each locus a simple-repeats and a AluY were N-masked. RNA45S can be subdivided

280 into the $5^{\prime}$ and $3^{\prime}$ external transcribed spacer (ETS), internal transcribed spacer (ITS) 1 and 2, and the mature rRNAs. Downstream of each RNA45S is a $29.6 \mathrm{~kb}$ intergenic spacer (IGS) which was not included in this study. B) Log-scaled coverage across hgrl in the CEPH-1463 trio. C) The cumulative distribution of intra-individual variant allele frequency of the 926 variant positions (see materials

285 and methods) for each member of the trio. The yellow vertical line at iVAF $=0.02$ is the threshold at which a variant was called as 'present' in an individual. D) The average distribution of variants (iVAF $>0.02$ ) in each region of hgrl. E) Evolutionary conservation (in Shannon Information bits(26)) of the mature rRNA variants relating to the intra-individual variant allele frequency. F) Detailed

290 coverage of NA12878 DNA- and poly-A selected RNA-seq, as well as K562 total RNA-seq at 28S.59G and 18S.1248T. The read coverage matching the reference sequence is colored in gray and mis-matching sequences are colored by the alternative base. E) The structure of i) uradine and ii) the hyper-modified nucleotide 1-methyl-3-( $\alpha$-amino- $\alpha$-carboxyl-propyl) Psuedouridine. 
bioRxiv preprint doi: https://doi org/10.1101/118760; this version posted March 21, 2017. The copyright holder for this preprint (which was not

A

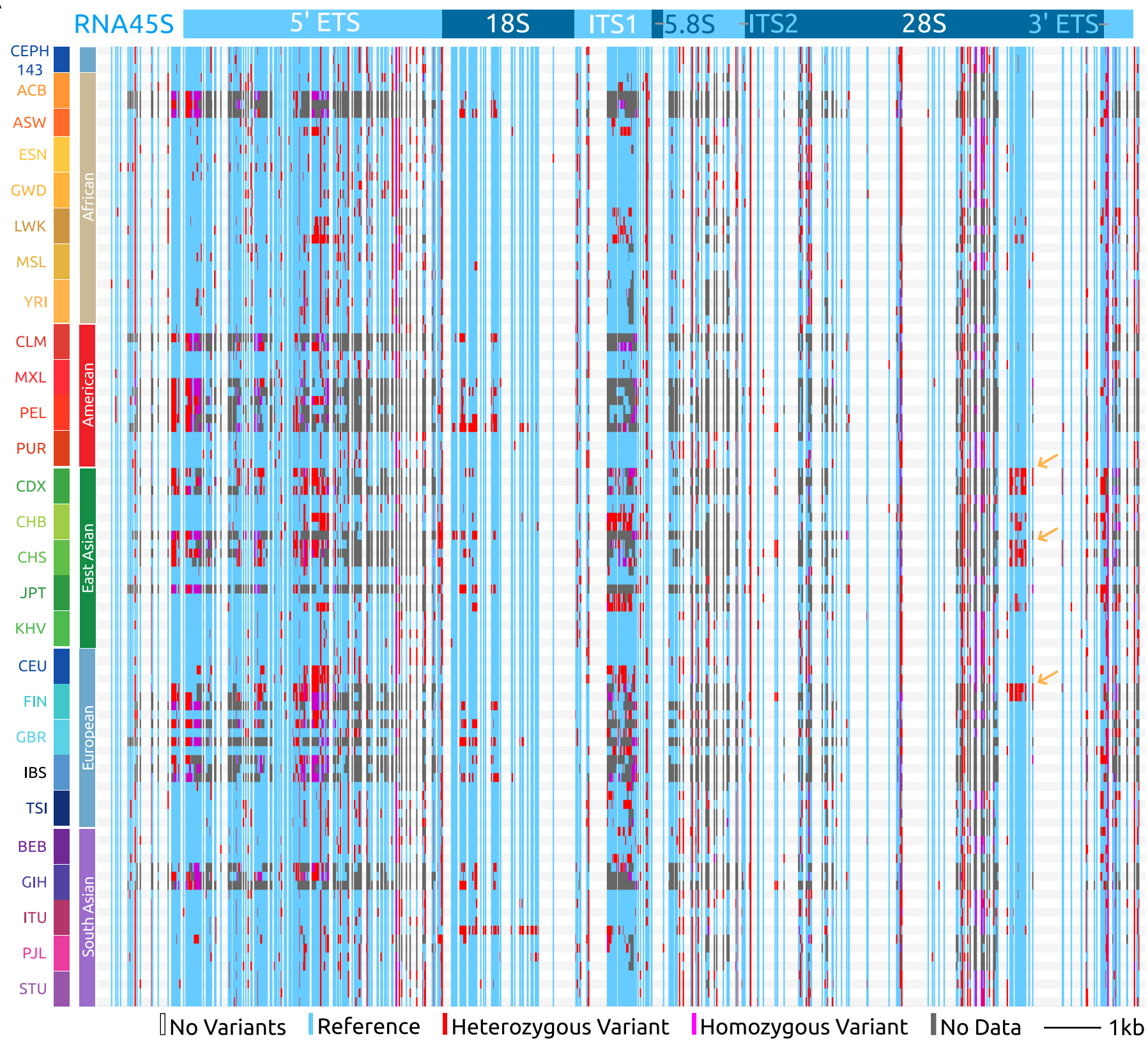

GC\% $0=100=\mathrm{ND}$

Bi.

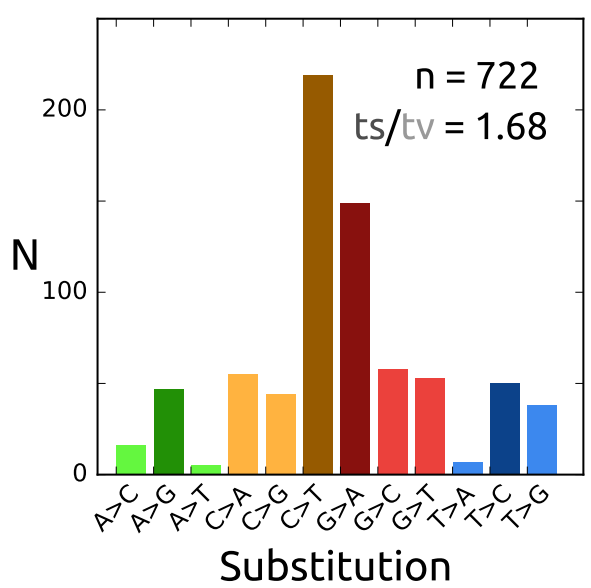

ii.

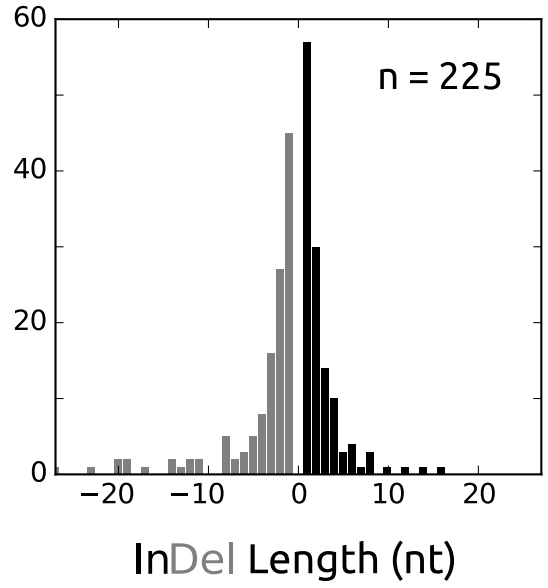

C

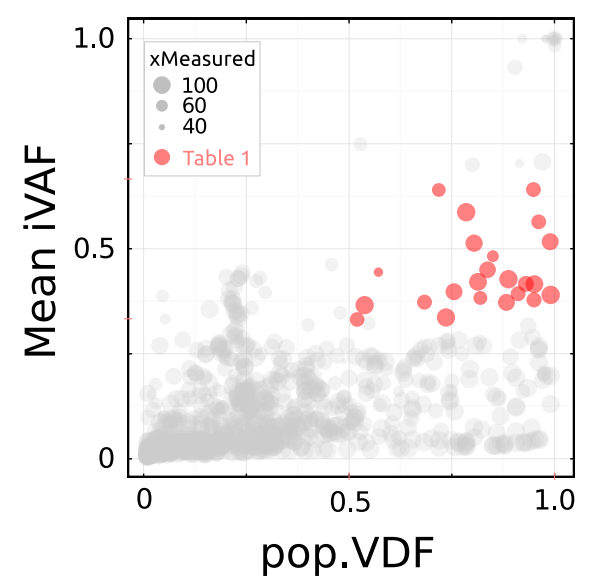




\section{Figure 2: Inter-individual variation at RNA45S}

A) Visualization of the high-quality variants from 107 individuals from 26 world populations. Vertical blue lines are sequences in which a variant genotype was called in at least one genome, with heterozygous and homozygous

300 genotyping in red and magenta respectively. Invariable positions are clear columns across the whole graph. Particular libraries in which there was no quality coverage at a variant is gray. Sequence GC\% is shown at the bottom in 30, 50 and 70 bp sliding windows. F) Transition vs. transversion nucleotide substitutions in the 722 single-nucleotide variants. E) Size distribution of the 225 indel variants.

305 D) Inter-individual, or population variant-detection frequency (pVDF) vs. Intraindividual variant allele frequency (iVAF) scaled by the number of distinct genomes in which the variant allele was measured (to account for regions of uneven read coverage in the data). Red highlighted variants have an average intra-individual allele frequency between $0.33-0.66$ and are found in at least half 310 of the genomes interrogated (see Table 1). 


\section{Supplementary Files}

Supplementary Table 1. Supplementary_Table1.xls

Sequencing datasets and their accessions used in this study.

Supplementary Sequence 1. hgrl.fa.gz

Hgrl reference rDNA sequence. Deposition into online nucleotide database pending.

Supplementary File 1: 107genomes.hgr1.g.vcf.gz

GVCF file of the 926 variants positions in the 107 genomes included in this study. 